\title{
Zweite Antwort auf den erneuten Angriff des Hrn. Prof. Dr. H. Senator in Berlin ${ }^{1}$ ).
}

(Als Beitrag zur thierischen Wärmelehre.)

$$
\text { Von }
$$

\section{E. Pflüger.}

Der in dem vorhergehenden Aufsatz enthaltene erneute Angriff Senator's enthält in so unklarer Fassung eine solche Menge von Irrthümern und Fntstellungen, dass ich wohl nochmals das Wort ergreifen muss.

Das Wesentliche in dem erneuten Angriff besteht darin, dass Senator seine ,Berichtigung" der Zahlen Colasanti's selbst als falsch zurückeiehen muss.

Er thut es aber in einer Fassung, die auf den nicht sehr aufmerksamen Leser den Eindruck machen wird, als ob es sich um einen bei der Controverse ganz unwesentlichen Punkt handle, weshalb er denn auch das Bèkenntniss, statt es an die Spitze seines Aufsatzes zu stellen, in eine sehr lange Anmerkung einfügt. Dieses Bekenntniss (S. pg. 496) lautet:

„,Ich habe in meiner ersten Berichtigung das Product von den Werthen der fiebernden Thiere abgesogen, anstatt sie $\approx u$ denen der gesunden wu addiren. Diess ist das Eineige (sic!), was Pflüger mit Recht rügt, da die Werthe, welche Colasanti gefunden hat, nur für das gesunde Thier gelten sollen. Die Consequenzen werden übrigens dadurch nicht geändert."

1) Siehe Senator, Noch ein Wort über Colasanti's "Beitrag etc. Dieses Archiv. Bd. 14. pg. 492. 
Zweite Antwort auf den erneuten Angriff d. Hrn. Prof. Dr. H. Senator. 503

Man sieht, Senator, der Colasanti einen logischen Fehler im Rechnungsansatz nachweisen und dessen Zahlen corrigiren wollte, muss zugeben, dass er sie unichtig corrigirt hat, dass seine "Berichtigung“ eine, Beumrichtigung" war. Das war natürlich ndas Einzige», was ich "mit Rechtuzu rügen hatte.

Aber wenn nun auch die Zahlen, welche die „Benurichtigung“" Senators ergab, falsch waren, so werden nach ihm ndie Consequenzen übrigens dadurch nicht geändertc. Die Steigerung der 0 xydationsprocesse im Fieber bleibt also auch jetzt noch bestehen - Senator zum Verdruss.

Statt nach dem ersten verunglückten Angriff sich ruhig zu verhalten, sucht er nun seine "Berichtigung « der Zahlen Colasanti's anders auszuführen, wobei er auf einem logischen Fehler verharrt, den er auch bei der ersten "Berichtigung " gemacht hat. Der zweite kritische Versuch Senator's ist deshalb ebenso haltlos, als der erste. Die Sache ist folgende.

Wie sich der Leser erinnert, hatte Colas a nti gefunden, dass ein fieberndes Meerschweinchen viel mehr Sauerstoff verbrauchte und Kohlensäure producirte als dasselbe Thier im gesunden Zustande. Als das Thier fieberte, war aber die Temperatur der Luft ein paar Grad niedriger. Da nun Gesunde bei niedrigerer Temperatur der Luft auch mehr Sauerstoff verbrauchen und Kohlensäure produciren, so entstand die Frage, ob der Mehrverbrauch des fiebernden Meerschweinchens schon durch die Abnahme der Temperatur der Luft allein erklärbar sei. Wir stellten also die Frage:

"Wie viel Sauerstoff würde dies Meerschweinchen, welches jetzt fiebert, verbrauchen, wenn es bei gleicher Temperatur der Luft nicht fiebernd, sondern gesund wäre?«

Diess liess sich mit hinreichender Genauigkeit auf Grund von Colasanti's zahlreichen Analysen berechnen, sodass dann die im Fieber und im gesunden Zustande constatirten Werthe des Gaswechsels unter einander vergleichbar wurden.

Wir stellten also fest, dass bei gleicher Temperatur der Umgebung die Oxydationsprocesse des fiebernden Meerschweinchens bedeutend energischer als die des gesunden gewesen seien.

Thatsachen festzustellen, kann doch nicht verboten sein - es fragt sich nur, ob man Schlüsse daraus zieht, die unberechtigt sind. Wir hatten aber gar keine Schlüsse daraus gezogen, sondern einfach die Thatsache gegeben. 
Nach Senator ist es aber ganz unexact, nach einer solchen Thatsache zu fragen, sondern ndie allein mögliche exacte Fragestellung « war folgende:

"Wie viel Sauerstoff würde dieses Meerschweinchen, welches jetzt fiebert, verbrauchen, wenn es bei gleichem Wärmeverlust d. h. bei gleicher Differenz zwischen seiner (Haut-) und der Um gebungs-Temperatur nicht fiebernd wäre?" (S.p. 493.)

Also wir verglichen den Stoffwechsel im fieberhaften und Normalzustande unter der Voraussetzung gleicher Temperatur der Umgebung; Senator hingegen unter der Voraussetzung gleichen Wärmeverlustes.

Es ist natürlich selbstverständlich, dass die Oxydationsprocesse des fiebernden Organismus mit denen des normalen unter der Voraussetzung verglichen werden sollten, dass der fiebernde Organismus nur diejenigen Unterschiede vom normalen darbietet, welche durch das Fieber wesentlich bedingt sind, während alle anderen Verhältnisse als möglichst gleichartig anzunehmen sind.

Ist nun aber nicht von jeher der Fiebernde mit dem Gesunden von dem Gesichtspunkte aus verglichen worden, ob er mehr Wärme producire als der Gesunde? Hat nicht Senator selbst zugeben müssen, dass im Fieber die Wärmeproduction gesteigert sei, dass also auch die Wärmeabgabe, der Wärmeverlust des Fiebernden an die Luft grösser sei, als bei dem Gesunden? Wenn also der Standpunkt für die Vergleichung des normalen und fieberhaften Organismus so genommen wird, dass der Fiebernde sich vom Gesunden, wie auch Senator zugibt, durch Mehrproduction von Wärme unterscheidet, so ist es klar, dass das Studium der Oxydationsprocesse von demselben Standpunkte aus unternommen werden muss. - Senator kann also logisch nicht verlangen, dass die Oxydationsprocesse Fiebernder und Gesunder unter der Voraussetzung gleichen Wärmeverlustes untersucht werden, ja diess als die mallein mögliche exacte Frage " bezeichnen. Wenn dauernd der Wärmeverlust des Fiebernden und Gesunden gleich sein soll, muss auch die Wärmeproduction gleich sein. Wenn man nun ein normales Thier unter solche erregende Bedingungen brächte, dass es eben so viel Wärme als ein fieberndes producirte, was Senator als exacte Vergleichung betrachtet, so wird dann ausser Sen a cor kein Sachverständiger erwarten, dass die Grösse der Oxydationsprocesse (bei gleich energischer Wärmebildung!) in beiden Fällen wesentliche Unterschiede darbietet. 
Ich will nun zeigen, durch welche logischen Fehler Senator zu der nach seiner Ansicht "allein möglichen exacten “ Fragestellung gelangt ist.

Sena to $x$ schwebt in Gedanken die Nothwendigkeit vor, die fieberhaften und normalen Organismen unter möglichst gleichen Bedingungen zu untersuchen. Ein fieberhaftes Thier, sagt sich Senator, verliert von seiner heissen Haut bei gleicher Temperatur der Umgebung mehr Wärne als ein gesundes Thier. Cojasanti hat aber gefunden, dass ein gesundes Meerschweinchen, welches mehr Wärme von seiner Haut verliert, deshalb einen energischeren Stoffwechsel hat als dasselbe Thier, wenn es weniger Wärme durch seine Haut an die Umgebung abgitt: Da also beim gesunden Thier die Oxydationsprocesse in dem Maasse, wachsen, als der Wämeverlust des Thieres wächst, folglich je nach der Grösse des Wärmeverlustes sehr verschieden sind, so muss man die Oxydationsprocesse des Fiebernden mit denen des Gesunden unter der Voraussetzung gleichen Wärmeverlustes vergleichen. Hiermit ist der Trugschluss vollzogen.

Senator hat nämlich micht beachtet, dass bei den Versuchen Colasanti's über Wärmeregulation das Thier sich bald in Luft von höherer, bald in solcher von niederer Temperatur befand und in Folge dessen bald mehr bald weniger Wärme verlieren musste. Wenn nun ein normales Thier sich in kühler Luft befindet, so bedingt der grössere Wärmeverlust durch die Haut erfahrungsmässig ein Sinken der Temperatur der Haut. Höchst wahrscheinlich reagiren nun die Hautnerven auf die Temperatur und werden um so stärker gereizt, bis zu dem lebhaftesten Schmerze, je niedriger die Temperatur der Haut wird. Je stärker aber diese Reizung der Hautnerven ist, desto energischer ist die reflectorische Anregung des Stoffwechsels durch sie. Bei sehr starker Abkühlung der Haut sehen wir ja reflectorisch alle Muskeln fortwährend zittern. Bereits 1875 entdeckte ich als Hinweis auf die bier in Betracht kommende Bedeutung des centialen Nervensystems, dass nach Durchschneiaung des Haismarks von Kaninchen dje Regulation der Körpertemperatur durch Variation der Wärmeproduction aufgeboben ist.

Senator hat also nicht beachtet, dass doch höchst wahrscheinlich die Grösse des Wärmeverlustes der Haut an sich gar keinen Einfluss auf den Stoffwechsel ausübt, sondern nur insofern und weil der Wärmeverlust die Temperatir der Haut herabsetzt. 
Wenn der verstärkte Wärmeverlust von der Haut zur Folge hat, dass die Temperatur der Haut bei constanter Innentemperatur des Organismus sinkt, so bedeutet diess, dass der Wärmevorrath des Körpers sinkt: eine Steigerung der Wärmeproduction ist in diesem Falle eine vernünftige Einrichtung.

Wenn aber der Wärmeverlust eines Thieres zunimmt und gleichzeitig trotzdem die Temperatur der Haut steigt, weil die Wärmeproduction etwa in Folge reichlicher Nahrung, starker Muskelarbeit gestiegen ist, oder weil eine künstliche Erwärmung der Haut durch heisse Umgebung (heisses Bad oder Luft) vorhergegangen, dann ist dieses Steigen der Temperatur der Haut ein Zeichen, dass der Wärmevorrath des Körpers zugenommen hat. Eine jetzt durch die Haut veranlasste Steigerung des Stoffwechsels, also weitere Steigerung des abnorm grossen Wärmevorrathes würde sinnlos und eine nutzlose Vergeudung von Kraft sein. Nein!

Die höher temperirte Haut erregt jetzt weniger energisch den Stoffwechsel, obwohl der Wärmeverlust ein sehr grosser sein kann.

Wenn also der gesteigerte Wärmeverlust eine Abnahme der Hauttemperatur erzeugt, so steigert diese den Stoffwechsel; wenn der gesteigerte Wärmeverlust aber mit einer Steigerung der Hauttemperatur verbunden ist, so nimmt die den Stoffwerhsel anregende Wirkung der Hautnerven ab. - Man muss also nicht mit S en a tor sich vorstellen, dass die Haut den Stoffwechsel um so stärker anrege, je grösser der Wärmeverlust der Haut an die Luft sei, welches eben nur so lange wahr ist, als dadurch die Temperatur der Haut abnimmt.

In Folge dieses Irrthums meinte dann Senator, dass, weil ein fieberndes Thier wegen seiner heissen Haut bei gleicher Temperatur der Luft mehr Wärme als ein normales verliere, liege in diesem grösseren Wärmeverluste des fiebernden Organismus eine Ursache stärkerer Erregung des Stoffwechsels als sie bei dem normalen Thiere vorhanden sei, das einen geringeren Wärmeverlust von seiner kühleren Haut erleide. Es ist aber gerade umgekehrt! Weil die Haut des Fiebernden wärmer ist, so liefert diese eine geringere Anregung des Stoffwechsels als bei dem Gesunden. Wenn man also die Mittel hätte, um die Anregung des Stoffwechsels, welche von der Haut aus veranlasst wird, bei Fiebernden und Gesunden gleich gross zu machen, so würde die Differenz der Oxydationsprocesse in beiden 


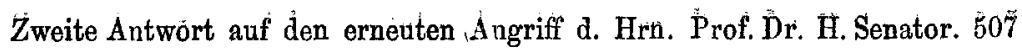

Zuständen, wie sie von Colasanti beobachtet wurde, sich nicht verkleinern, sondern vergrössern.

In Folge seines Irrthums will ja Senator durch seine $\# \mathrm{Be}-$ richtigung « diese Differenz verkleinern.

Vergleicht man also die Oxydationsprocesse Fiebernder und Gesunder unter der von uns gemachten Voraussetzung gleicher Temperatur der Umgebung, so ist die fieberhafte Steigerung der Oxydationsprocesse, wenn sie thatsächlịch nachgewiesen wird, sogar a fortiori bewiesen.

Hiermit ist die neue Correctur Senator's widerlegt.

Ich hatte in meiner ersten Beantwortung der "Berichtigung" Senator's schon gezeigt, dass - unter einer bestimmten berechtigten Voraussetzung - Colasa nti's Zahlen für normale und fieberhafte Zustände unmittelbar vergleichbar seien und keiner Correctur bedürften.

Die Luft war allerdings $z u$ der Zeit als das Thier fieberte ein paar Grad niedriger (und zwar: 3,80 $\mathrm{C}$. in maximo) als zu der Zeit, wo es gesund war. Da aber Colasanti durch einen directen Versuch gezeigt hatte, dass sogar eine Abnahme der Lufttemperatur um $10^{\circ} \mathrm{C}$., sage $10^{\circ} \mathrm{C}$., keine Veränderung der Oxydationsprocesse desselben fiebernden Thieres erzeugte (Siehe Versuch 7 u. 8. pg. 471. dieser Band), so konnte man mit sehr grosser Wahrscheinlichkeit annehmen, dass die geringe Temperaturveränderung, welche die Luft seit dem Krankwerden des Thieres erlitten hatte, nicht die Ursache der Steigerung der Oxydationsprocesse sein könne, dass diese Ursache vielmehr im Fieber liege. Um diese Veränderung der Lufttemperatur und ihre mögliche Beeinflussung des Stoffwechsels dreht sich wesentlich der ganze Streit. Senator ignorirt in seinem zweiten Angriff diesen von mir so eben und schon früher deutlich entwickelten Thatbestand und erregt dadurch den Anschein, als ob er ignorire, weil er durchaus uns mberichtigen " will.

Damit der Leser spätere Erörterungen leicht zu verstehen im Stande sei, will ich hier denselben auf den merkwürdigen Umstand 
aufmerksam machen, dass Senator, um seine "Berichtigung " an Colasanti's Zahlen anbringen zu können, die Voraussetzung machen muss, dass die Körpertemperatur der Warmblüter ebensowohl durch Regulation der Production als des Verlustes der Wärme constant erhalten werde. Nun läugnet ja aber bekanntlich Senator principiell die Regulation der Temperatur des lebendigen Körpers durch Variation der Wärmeproduction. Wie dies Dilemma zu lösen ist, dariber lässt er sich in folgendem Satz (pg. 496) aus, dessen Sinn ich sicher zu ergründen nicht vermocht habe:

"Ausdrücklich aber bemerke ich, dass ich die Correctur nur in dem Sinne für zulässig halte, in welchem Pflüger und $\mathrm{Co}_{0}$ las anti ihre anderen an gesunden Thieren bei verschiedener Aussentemperatur angestellten Versuche auffassen, dass ich aber die Berechtigung dieser Auffassung, wonach für je $1^{0}$ Temperaturabnahme die Gasmengen um ein Bestimmtes steigen sollen; damit nicht anerkenne. Die Versuche Colasanti's beweisen das nicht, was aus ihnen geschlossen wird, wie ich bei einer anderen Gelegenheit zeigen werde".

Soll dieser Passus einen Sinn haben, so kann er nur bedeuten, dass $\mathrm{S}$ e $\mathrm{n}$ a tor in seinen "Berichtigungen « darauf aufmerksam machen will, auf welche Art logisch und correct die Zahlen Colasanti's vergleichbar gemacht werden müssen, wenn man die nach Senator falsche Voraussetzung zulässt, dass die Oxydationsprocesse der Warmblüter wirklich mit abnehmender Temperatur der Luft zunehmen.

Was soll dann aber seine Correctur, wenn sie nach seiner eigenen Ansicht wegen falscher Prämisse doch illusorisch ist? Die Rechnung Colasanti's erscheint dann als das Phantom, an dem Senator Logik tractirt.

Ich gehe nun zu der Betrachtung verschiedener anderer in dem neuen Angriff enthaltener Irrthümer über, die mit der ersten und zweiten Berichtigung der Zahlen Colasanti's in einer wesentlichen Beziehung stehen.

Die folgenden Erörterungen werden den Leser von der Vorliebe Senator's überzeugen, in vielen von Colasanti und mir mitgetheilten Thatsachen und Ansichten, welche mit den Theorieen 
Senator's unverträglich sind, höchst erfreuliche Bestätigungen seiner Lieblingstheorieen zu erkennen.

Senator behauptet also, dass Colasanti's Beobachtung der stark gesteigerten Kohlensäureausscheidung des fiebernden Meerschweinchens seinen Lieblingstheorieen "nicht ungünstig, sondern eher noch im böchsten Grade günstig “ sei (pg. 497). Denn er (Senator) habe gelehrt ${ }^{1}$ ): "dass die fieberhaft vermehrte Ausscheidung von Kohlensäure entweder ganz oder zum allergrössten Theil durch die günstigeren Ausscheidungsbedingungen verursacht sein muss “ (pg. 498).

An derselben Stelle hebt er noch besonders hervor, "dass noch gar kein zwingender Grund vorliegt, die fieberhaft vermehrte Ausscheidung von Kohlensäure auf eine vermehrte Bildung derselben im Thierkörper zurückzuführen « ${ }^{2}$ ).

Da nach allgemeiner Ansicht die Kohlensäurebildung die Hauptmenge der thierischen Wärme erzeugt und die Kohlensäureexhalation im Fieber bedeutend auch nach Senator vermehrt ist, so ist die auch nach Senator stark vermehrte Wärmeproduction im Fieber nach $\mathrm{S}$ en ator am natürlichsten aus unbekannten Ursachen zu erklären!!!!!!, wobei dann nach Senator das Eindringen von Explosionskörpern in den Organismus und andere pikante Einfälle zu discutiren sind ${ }^{3}$ ).

Colasanti's Versuch beweist aber eine vermehrte Oxydation im Fieber unfehlbar und hebt deshalb die Berechtigung auf, an der vermehrten Bildung der Kohlensäure im Fieber zu zweifeln, macht also Senator's Lieblingstheorie von der nur besseren Abscheidung der bereits präexistirenden Kohlensäure im Fieber unmöglich. Der Beweis Colasanti's liegt darin, dass die Sauerstoffabsorption ebenfalls im Fieber ausserordentlich and entsprechend der stärkeren Kohlensäureausscheidung gesteigert ist. Ich habe mit meinen Schülern Dr. Finkler und Dr. Oertmann den Beweis geliefert, dass die stärksten Veränderungen der Athembewegungen, die bedeutendsten ohne Erstickungszufälle erträglichen Veränderungen des Blutkreislaufs keine Spur eines Einflusses auf die Sauerstoffabsorption der Thiere ausüben. Kein Satz der Physiologie ist sicherer, als mein Gesetz, dass jede, auch nur 20 Minuten anhaltende, mit Sicher-

1) Sen a tor, Untersuchungen'über den fieberhaften Process 1873. pg. 73.

2) Senator, a. a. 0. pg. 73 .

3) Senator, a. a. 0. pg. 141 u. ff. 


\section{heit constatirte Steigerung der Sauerstoffabsorption eine Steige- rung der 0xydationsprocesse beweist.}

Da nun bei den Fieberversuchen Colasanti's, die an demselben Thier an verschiedenen Tagen und zu verschiedenen, zufällig herausgegriffenen, Tageszeiten angestellt wurden, jedesmal die Sauerstoffabsorption sehr bedeutend gesteigert war und ganz analog die Kohlensäureausscheidung, so ist es nun einmal so gewiss wie der Pythagoräische Lehrsatz, dass bei diesem fiebernden Meerschweinchen ein gesteigerter Verbrennungsprocess vorhanden war. Es ist unzweifelhaft: die Sauerstoff bestimmungen Colas anti's bringen den Beweis für die bedeutende Steigerung der Oxydationsprocesse im Fieber, das nicht einmal sehr stark war. $\left(39,7^{\circ}\right.$ C. in Recto.)

Senator wagt nun zu sagen:

"S auerst off bestimmungen habe ich selbst nicht gemacht, Pflüger's und Colasanti's Befunde könnten demnach in dieser Beziehung auf den ersten Blick weder günstig noch ungünstig für meine "Lieblingstheorieen" erscheinen «. (Dieser Bd. pg. 498.)

Also weil wir nicht Senator durch die Bestimmung der Sauerstoffabsorption des fiebernden Thieres, welche auf keine andere Weise als durch den Oxydationsprocess gesteigert werden kann, seiner Lieblingstheorie der im Fieber nicht vorhandenen Mehrproduction der Kohlensäure einen Riegel vorgeschoben haben, sollen diese Bestimmungen des Sauerstoff jenen Theorieen "weder günstig noch ungünstig«, also irrelevant sein? Es kommt mir fast wie Scherz vor!

Ein anderes Bild:

Bekannt ist das Theorem Senator's ${ }^{1}$ ):

"Bis jetzt ist nach allen vorliegenden Thatsachen im Fieber keine Uebereinstimmung zwischen Wärmehaushalt und Stoffumsatz nachweisbar".

Der eigentliche Sinn dieses Satzes ist nämlich, dass weil nach Senator im Fieber im allergünstigsten Falle eine Steigerung der Kohlensäurebildung von nur $30-40$ Procent ${ }^{2}$ ) angenommen werden könne, wahrscheinlich aber gar keine Mehrbildung von Koh-

1) Senator, Unters, über d. fieberhaft. Process. p. 89.

2) Senator, Unters, über d. fieberhaft. Process. pg. 166. Thesis 4. 
lensäure stattfinde, - die factisch erzeugte Wärme unerklärlich sei. Nun verzeichnet Leyden ${ }^{1}$ ) aber auch Steigerungen der febrilen Kohlensäureausscheidung bis 40, ja sogar 70 Procent. Mit Rücksicht auf die Fieberwärme aber sagt Senator ${ }^{2}$ ): "Man kann danach die durchschnittliche Zunahme des Wärmeverlustes während der Fieberhitze im Vergleich zur Norm auf etwa 70-75 Procent veranschlagen «.

Das Theorem Senator's der Unerklärlichkeit der Fieberwärme aus den Oxydationsprocessen ist desshalb schon ganz bodenlos, weil bis dahin über den Sauerstoffverbrauch im Fieber Nichts bekannt ist. Die thierische Wärme entsteht nach Allem, was wir wissen, wesentlich dadurch, dass der eingeathmete Sauerstoff sich mit dem Kohlenstoff des Organismus zu Kohlensäure, mit dem Wasserstoff zu Wasser chemisch verbindet. Nur wenn man bei Unkenntniss der verbrennenden Moleküle den Sauerstoffverbrauch und die Kohlensäureausscheidung kennt, vermag man die mögliche Menge von Wasserstoff zu berechnen, welche oxydirt wird. Wenn bei Nahrungsentziehung der gesunde Organismus gezwungen wird, sowie es im Fieber geschieht, auf Kosten seiner eignen Substanz zu leben, dann wird $1 / 4$ bis $1 / 3$ des eingeathmeten Sauerstoffs zur Oxydation des Wasserstoffs verbraucht ${ }^{3}$ ). Nun wäre es doch möglich, dass bei der gesteigerten Fiebertemperatur, durch welche der Organismus wahrscheinlich schädliche Bestandtheile und Fermente zur Oxydation bringt und so durch Feuer reinigend heilt (Siehe meine Abhandlung über die möglichen Maximaltemperaturen des Organismus ${ }^{4}$ ) ), kohlenstoffreichere und wasserstoffärmere Stoffe - etwa Derivate des Benzols entstehen. - In diesem Falle könnte im Fieber ein noch grösserer Theil des absorbirten Sauerstoffs zur Oxydation von Wasserstoff verbraucht werden. Leider sind die Versuche $\mathrm{C}_{0}$ Iasanti's zu wenig zahlreich, um zu allgemeinen Schlüssen $\mathrm{zu}$

1) Leyden, Deutsches Archiv f. klin. Med. VII. 536.

2) Senator, Unters. über d. fieberh. Proc. pg. 139.

3) Regnault und Reiset. Ann. d. Chim. Phys. T. 26.

R. u. R. fanden als Werth des sespiratorischen Quotienten $\alpha$

$\begin{array}{llll}\text { bei nüchternen Kaninchen: } & 0,690 & \text { (pg. 407 l. c.) } \\ \text { bei nüchternen Hunden: } & 0,724 & \text { (pg. } 428, \text { ) } \\ \text { bei nüchternen Hühnern: } & 0,662 & \text { (pg. } 461, \text { ). } \\ \text { Pflü ger, Dieses Archiv } & \text { Bd. 10. }\end{array}$

4) E. Pflüger, Dieses Archiv Bd. 10. pg. 641. 
berechtigen mit Rücksicht auf die Art der Verwendung des Sauerstoffs im fieberhaften Organismus.

Wahrscheinlich aber wird ein beträchtliches Quantum des absorbirten Sauerstoffs im fiebernden Organismus zur Oxydation von Wasserstoff verwandt, wobei noch in Betracht kommt, dass eine bestimmte Menge Sauerstoff mehr Wärme erzeugt, wenn sie zur Oxydation von Wasserstoff statt von Kohlenstoff verbraucht wird.

Aber selbst die Kenntniss des absorbirten Sauerstoffs und der producirten Kohlensäure reicht nur zu einer ungefähren Berechnung der Wärmeproduction aus, da dieselben Mengen von Wasserstoff und Kohlenstoff bei Oxydation zu Wasser und Kohlensäure verschiedene Wärmemengen liefern je nach der chemischen Natur der Verbindungen, in denen sie enthalten waren. Die im Fieber der Oxydation vorzugsweise unterliegenden Bestandtheile des Organismus sind uns aber unbekannt.

Dass auch die Kenntniss der producirten Harnstoffmenge keinen Schluss auf Natur und Grösse der Oxydationsprocesse gestattet, bedarf keiner besonderen Begründung.

Erwägt man nun noch die Unsicherheit in der Bestimmung der factisch in einem Tage von einem Fiebernden producirten Wärmemengen, so sieht man, dass jede thatsächliche exacte Grundlage zur Ausführung einer Wärmebilanz fehlt.

S enator handelt wie ein Kaufmann, der über den Zustand eines Vermögens ein Urtheil fällt, obwohl er die Passiva unsicher und einen grossen Theil der Activa gar nicht kennt.

Vor der Hand ist also Senator nicht berechtigt zu der Behauptung, dass die Oxydationsprocesse im Fieber die factische Wärmeproduction nicht erklären. Da aber diese Behauptung höchst wahrscheinlich grundfalsch ist, weil sie allen anderen Kenntnissen in der thierischen Wärmeökonomie widerspricht, so sollte man einen solchen Satz gar nicht aufstellen.

Wodurch hat nun also Colasanti eine Bestätigung des Paradoxon's von Senator geliefert? Sie soll in Versuch 7 und 8 liegen (S. pg. 471 dieses Bandes).

Um den Irrthum Senator's leicht zu begreifen, muss ich einige Bemerkungen über die Natur der Schwankung des »respiratorischen Quotienten « machen.

Bekanntlich vollzieht nach den Erfahrungen aller Beobachter der $\gg$ respiratorische Quotient $«$ bei demselben Thiere unter scheinbar 
denselben Bedingungen periodische Schwankungen; d. h. Sauerstoffabsorption und Kohlensäureexhalation ändern sich im Allgemeinen gleichzeitig nicht in absolut derselben Proportion. Da beide Werthe der Regel nach gleichzeitig wachsen und gleichzeitig abnehmen, so ist es selbstverständlich, dass es immer Zeiträume geben wird, in denen der eine Werth noch sinkt, während der andere schon wieder zu steigen anfängt.

Weil in Versuch 7 und 8 Colasanti's bei gleich starkem Fieber dieses Verhältniss vorliegt, dass in Versuch 8 der Sauerstoffverbrauch eine Spur kleiner, die Kohlensäure aber etwas grösser als in Versuch 7; vielleicht gar, weil das Thier wegen der niederen Temperatur der Luft in diesem Versuch ein weniger tiefer athmete, schliesst Senator: dass in beiden Füllen ein ,entgegengesetztes Verhalten des Stoffwechsels" im Fieber vorgelegen; dass also „im Fieber keine Uebereinstimmung zwischen Wärmehaushalt und Stoffumsatz nachweisbar" sei. (S. pg. 499.)

Die Wirklichkeit ist die, dass in Versuch 7 und 8 bei gleich starkem Fieber der Stoffwechsel fast gleich stark war, weil die $\mathbf{A b}$ weichungen in Sauerstoff und Kohlensäure sogar bedeutend kleiner sind als sie unter denselben Bedingungen bei gesunden Thieren beobachtet werden. (Siehe als Beleg Colasanti's Versuch 16.17. 18.)

Die Zahlen sind:

\begin{tabular}{l|c|c} 
& Sazerstoff: & Kohlensäure: \\
Vers. 7 & 1137.3 Cc. & 949.5 \\
Vers. 8 & 1119.1, & 1017.1
\end{tabular}

Die Steigerung der Kohlensäure von Versuch 7 zu Versuch 8 beträgt $7,1 \%$. Wir haben oben gesehen, dass Steigerungen der Kohlensäureausscheidung um $30-40 \%$ nach Senator im Fieber nichts beweisen für eine Steigerung der Bildung; hier aber, wo seine Lieblingstheorie ihm wieder andere Wünsche eingibt, benutzt er eine zufällige in den normalen Schwankungen liegende nur $7,1 \%$ betragende Steigerung, um auf den Stoffwechsel zu schliessen.

Wer in aller Welt kann überhaupt aus diesen irrelevanten Abweichungen eines einzigen Beispiels schliessen, dass der Stoffwechsel in Versuch 8 ein "entgegengesetztes Verhalten " gezeigt wie in Versuch 7, ja als Argument für Sen at or's Paradoxon verwerthen wollen? Gerade das Verhalten des Sauerstoffs verbietet hier auf eine Steigerung der Kohlensäureproduction zu schliessen. 
Senator erschliesst noch eine andere merkwürdige Bestätigung einer anderen Lieblingstheorie aus Colas an ti's Fieberversuch durch folgende Dialektik:

Pflüger und Colasanti vermuthen: im Fieber sei die Regulation der Körpertemperatur durch Variation der Wärmeproduction nahezu aufgehoben;

Senator lehrt 1): es gibt principiell überhaupt gar keine solche Regulation - weder im gesunden noch kranken Zustande;

Also haben Pflüger und Colasanti Senator's Lieblingstheorie bestätigt; denn sie vermu then (!) ja auch, dass es wenigstens im Fieber fast keine Temperaturregulation gebe. - P flüg er und Colasanti meinen zwar die Regulation durch Production, Senator die durch Variation des Verlustes der Wärme - einerlei! es ist nach Senator Uebereinstimmung. Ich bitte den Leser nochmals die Thesis III. der Anmerkung zu lesen.

1) Drei Stellen aus Senator's Schriften zum Beleg:

I.

DNach alledem scheint es mir unzweifelhaft zu sein, dass die Aenderungen der umgebenden Temperatur auf die Wärmeproduction ohne Einfluss sind und dass es also eine besondere unwillkürliche Regulirung der letzteren je nach dem Wärmeverlust nicht (!!) gibt. S Senator, Beiträge zur Lehre von der Eigenwärme und dem Fieber. Virchow's Archiv. Bd. 45. pg. 366.

II.

In einer späteren über das Verhalten der Körperwärme bei Abkühlung der Haut 1870 publicirten Abhandlung sagt er:

„Schliesslich und hier berühren wir die wichtigste, weil practische Seite der Frage, ist es die therapentische Wirkung der Abkïhlungen, welche mit der Annahme einer während derselben stattfindenden Vermehrung der Wärmeproduction nicht (!!) zu vereinigen ist. (Se nator in Virch. Arch. Bd. 50. p. 370.)

III.

Mit Rücksicht auf das Fieber sagt S e n a tor (Virch. Arch. Bd. 45 pg. 370):

${ }$ Nach der in letzter Zeit am meisten zur Geltung gekommenen Ansicht soll, wie ich schon oben angedeutet habe, das Wesen des Fiebers in einer Störung des angenommenen (!!) besonderen Wärmeregulationsapparates beruhen, ohne welche weder vermehrte Wärmeproduction, noch verminderter Wärmeverlust ( $T r a u b e$ ) das Fieber erklären könnte. Da diese eigenthümliche Wärmeregulation nicht vorhanden ist, so kann auch von einer Störung derselben nicht die Rede sein. 
Senator stellt uns in seinem letzten Angriff eine weitere Kritik der anderen Arbeit Colas anti's in Aussicht, obwohl sich darin der definitive Beweis findet, dass die Temperatur der Warmblïter nicht bloss durch Regulation des Wärmeverlustes, sonder auch der Wärmeproduction constant erhalten wird. Man begreift wohl, wie unangenehm diese Arbeit Colasanti's unserem Kritiker sein mag, nachdem er durch Jahre lange Untersuchungen über Wärmeproduction,. Temperaturtopographie, Respiration und Stoffwechsel in zahlreichen Abhandlungen das Resultat niedergelegt hat, dass diese grossartige Natureinrichtung principiell nicht existire. Diese wohlthätige Einrichtung, die das Leben der Thiere und des Menschen in der Kälte des Winters schirmt und den Körper erwärmt, existirt und wird trotz aller früheren und zukünftigen Abhandlungen Senator's existiren im Glauben der Wissenschaft auf ewige Zeiten.

Die zukünftigen Abhandlungen Senator's sollen aber nach seiner Schlusserklärung (S. den vorhergehenden Aufsatz) nicht mehr in diesem Archive erscheinen. Senator wird es zuzuschreiben sein, wenn dann meine Antworten seinen Aufsätzen nicht mehr unmittelbar nachfolgen und die so wünschenswerthe sofortige umfassendere Aufklärung des Lesers über Senator's und meine Gedanken nicht mehr möglich ist. Senator muss mir selbst bezeugen, dass ich der Aufnahme seiner Abhandlungen nicht die kleinste Schwierigkeit bereitet, sondern sofort nach Einlaufen sein Manuscript zum Satz gebracht und sogar die Correcturen ihm schleunigst geschickt worden sind. Die Revision seiner Correcturen ist von mir selbst ansgeführt, damit der Text genau mit Senator's Intensionen übereinstimme.

Um meinerseits keine Ursache zu weiterer Verwirrung zu geben, betone ich, dass ich Alles, was in meiner ersten Antwort stand, absolut aufrecht erhalte, wenn ich auch nicht Alles nochmals besprochen habe, da ich ohnehin dem Angriff Senator's mehr als hinreichende Berücksichtigung gewährte. - Die Vergleichung der Oxydationsprocesse im fieberhaften und Normalzustande ist also auch in dieser Abhandlung unter der Voraussetzung gemacht, dass die Temperatur der Umgebung eine mittlere oder diese um einige Grade nach aufwärts überschrejtende sei, weil diess der für die Beurtheilung der Verhältnisse am Menschen zunächst wichtigste Gesichtspunkt ist. 
Nur eine persönliche Bemerkung zu machen habe ich noch die Pflicht. Senator sagt in dem vorhergehenden Aufsatze:

"Pflüger deutet an in Worten (1. c. p. 451), welche wiederzugeben mir nicht ziemt, dass ich die Berichtigung Colasanti's unternommen hätte in der Absicht den wirklichen Sachverhalt zu entstellen und Thatsachen, welche meinen $\gg$ Lieblingstheorieen $*$ nicht günstig sind, aus der Welt zu schaffen. Die Unterstellung solcher Beweggründe ist nicht Gegenstand einer wissenschaftlichen Erörterung, ich lasse sie unberührt.*

Diese Anklage Senator's ist eine Unwahrheit.

Meine Worte waren:

"Trotz der von Dr. Colas anti in dieser Publication beobachteten bescheidenen Zurückhaltung findet sich Senator veranlasst, einige "berichtigende c Correcturen an Colasanti's Zahlen anzabringen, wodurch die während des Fiebers beobachtete scheinbare Vergrösserung des Stoffwechsels nicht etwa eliminirt, sondern nur etwas verkleinert wird, sodass dieselbe noch immer $10 \%$ beträgt, wobei doch auch noch in Betracht kommt, dass es sich um kein sehr starkes Fieber handelte, da die Temperatur im Mastdarm nicht über 39,7 C. betrug. Um nun jene $10 \%$ Steigerung des Stoffwechsels, die also sogar von Senator zugegeben werden muss, in ihrer Bedeutung noch mehr herabzudrücken, sagt er, dass beĩ diesem fiebernden Meerschweinchen die Sauerstoffaufnahme $\rightarrow u m$ ein Geringes vermehrt war, als ob die Variation eines Naturwerthes um 10\% von irgend einem Naturforscher für etwas $>$ Geringes « angesehen werden könnte; - und als ob ein Forscher die Wucht unläugbarer Thatsachen, die seinen Lieblingstheorieen ungünstig sind, dadurch aus der Welt schaffen könnte, dass er wie der Vogel Strauss seinen Kopf in den Sand steckt."

Diese Worte enthalten keine Beleidigung. - Denn der Vogel Strauss steckt ja den Kopf nicht aus einer unlauteren oder böswilligen Absicht in den Stand, sondern weil er in der Aufregung und Angst die Verhältnisse unrichtig beurtheilt und glaubt, dass die Gefahr nicht existire und aus der Welt geschafft sei, wenn er sie nicht sähe.

Wahrscheinlich findet Senator die Beleidigung besonders in dem Passus von mir:

$\rightarrow \mathrm{Um}$ nun jene 10\% Steigerung des Stoffwechsels - - in ihrer Bedeutung noch mehr herabzudrücken, sagt er (S e nator), 
dass bei diesem fiebernden Meerschweinchen die Sauerstoffaufnahme >um ein Geringes« vermehrt war. «

Wenn Einer die Bedeutung einer Thatsache absichtlich herabzudrücken sucht, weil sie nach seiner ehrlichen aber irrigen Ansicht viel weniger belangreich ist, als das doch in Wirklichkeit der Fall ist, so begeht er einen Irrthum, aber nichts Unehrenhaftes.

Zum Verständniss von Senators Polemik wird schliesslich die historische Bemerkung nicht bedeutungslos sein, dase er als junger Mann dem wissenschaftlichen Einflusse des Prof. L. Traube in Berlin sich hingab und sich dann in seinen Abhandlungen für dessen die Wärmeregulation und das Fieber betreffende Theorieen engagirte, in denen jener Gelehrte sich durchaus geirrt hatte. Der Fortschritt der Wissenschaft hat bei fortwährendem Widerspruch und gleichzeitigem Zurückweichen Senator's jene Theorieen Stück um Stück zertrümmert und Colas anti und ich haben zuletzt zur Klärung der Lehre von der Wärmeregulation noch ein nachdrückliches Stück Arbeit gethan.

Ohne provocirt worden zu sein und zu einem sachlich ganz irrelevanten Zweck hat Senator nach dem ersten, wie er selbst theilweise gesteht, verunglückten Angriff, gegen mich und Colasanti einen zweiten unternommen, wie er schlechter begründet und in jeder Hinsicht fehlerhafter gar nicht möglich ist; ausserdem aber weitere Angriffe in Aussicht gestellt. Er hat bei dieser Polemik eine so beispiellose Verwirrung angerichtet, dass ich die Zeit nur bedauern kann, welche ich zur Entwirrung und Widerlegung fast nutzlos verloren habe. 